\title{
Crosslinkable Potato Starch-based Graft Copolymer Emulsion for Humidity Controlling Coatings
}

\author{
Rong-Min Wang ${ }^{\mathrm{a}, \mathrm{b} *}$, Xiao-Wen Wang ${ }^{\mathrm{a}, \mathrm{b}}$, Jun-Feng Guo ${ }^{\mathrm{a}, \mathrm{b}}$, Yu-Feng He ${ }^{\mathrm{a}, \mathrm{b}}$, Meng-Lan Jiang ${ }^{\mathrm{a}, \mathrm{b}}$ \\ ${ }^{a}$ Key Laboratory of Eco-Environment-Related Polymer Materials of Ministry of Education, \\ College of Chemistry \& Chemical Engineering, Northwest Normal University - NWNU, \\ 730070, Lanzhou, Gansu, China \\ ${ }^{\mathrm{b}}$ Key Laboratory of Polymer Materials of Gansu Province, College of Chemistry \& Chemical \\ Engineering, Northwest Normal University - NWNU, 730070, Lanzhou, Gansu, China
}

Received: October 23, 2012; Revised: April 19, 2013

\begin{abstract}
A new kind of crosslinkable potato starch-based graft copolymer emulsion (PoSt-g-BMD) has been prepared using butyl acrylate (BA) and methyl methacrylate (MMA) as main monomers, and diacetone acrylamide (DAAM) as functional monomer. The keto-carbonyl group of DAAM in PoSt-g-BMD copolymer could react with adipic dihydrazide $(\mathrm{ADH})$ at ambient temperature to form cross-linked copolymer. The PoSt-g-BMD had been characterized by the FT-IR spectra and thermogravimetric analysis (TGA). It was applied to prepare the humidity-controlling coatings (PoSt-g-BMD-C). The water absorption of PoSt-g-BMD-C was $105 \%$. The humidity controlling properties were also measured, and the PoSt-g-BMD-C exhibited humidity sensitivity and humidity retention because fillers of porous structure combined with PoSt-g-BMD copolymer emulsion based on scanning electron microscopy images (SEM). It could be widely applied in indoor coating for controlling humidity.
\end{abstract}

Keywords: potato starch-based copolymer, ambient cross-linked copolymer, graft copolymerization, humidity-controlling coating

\section{Introduction}

In order to achieve a healthy and comfortable indoor environment, it is very important to control humidity level. Many evidences indicate that indoor humidity environment is closely related to health problems and it also affects energy consumption and the durability of the building envelope ${ }^{1}$. The humidity controlling materials that absorb and release moisture can be used positively to reduce the extreme values of humidity levels in indoor climates ${ }^{2,3}$. The humidity-controlling coatings are one of the humiditycontrolling materials and are different from the conventional coatings. They must have strong absorption and good water retention, which means faster response for water ${ }^{4-7}$. The humidity-controlling coatings are composed of the binder, porous or more layers of filler and pigment ${ }^{8}$. However, almost all binders of humidity-controlling coatings were synthetic polymers that hardly decompose in the nature.

Starch, a kind of naturally abundant polysaccharide from a great variety of crops, has been used as not only food but as a kind of low cost biodegradable polymer material. Yet, the properties of starch-based materials are often responsive to the changes of the environment humidity ${ }^{9}$, temperature or $\mathrm{pH}^{10}$. In order to improve properties or expand its application field, many chemical modifications ${ }^{11-17}$ of starch have been carried out through hydroxyl groups. Particularly, graft copolymerization on $\operatorname{starch}^{18-22}$ gives the possibility to synthesize new highly added value materials for various applications, which were applied for the textiles, composites,

*e-mail: wangrm@nwnu.edu.cn keeping the variation in the indoor climate and $\mathrm{pH}$-sensitive starch hydrogels, respectively.

In this paper, A new kind of crosslinkable PoSt-based graft copolymer (PoSt-g-BMD) was prepared via graft polymerization. The keto-carbonyl group of DAAM in PoSt-g-BMD copolymer could react with adipic dihydrazide $(\mathrm{ADH})$ at ambient temperature to form cross-linked copolymer. The PoSt-g-BMD had been characterized by the FT-IR spectra and thermogravimetric analysis (TGA). It was applied to prepare the humidity-controlling coatings (PoSt-g-BMD-C). The water absorption and humidity controlling function of the coatings were also investigated, and the PoSt-g-BMD-C exhibited humidity sensitivity and humidity retention.

\section{Experimental}

\subsection{Materials}

Butyl acrylate (BA) and methyl methacrylate (MMA) were obtained from commercial sources. They were washed with dilute $\mathrm{NaOH}$ solution and deionized water to remove inhibitors, then dried with desiccant, respectively. All the purified monomers were stored at $-2{ }^{\circ} \mathrm{C}$ before use. The potato starch (PoSt) was obtained from Lanzhou Shuangshuang Chemical Industry Co.. Diacetone acrylamide (DAAM), adipic dihydrazides (ADH), and potassium persulfate (KPS) were received analytical without further purification. Titanium dioxide, talc, montmorillonite, kaolin, 
bentonite, diatomite (CD02), diatomite (CD05), natural crystalline calcium carbonate and precipitated calcium carbonate were all industrial grade.

\subsection{Preparation of crosslinkedable PoSt graft copolymer (PoSt-g-BMD)}

PoSt and water was added to gelatinization in a fourneck flask equipped with a thermometer, a condenser, a mechanical stirrer, dropping funnels for 0.5 hour at $85^{\circ} \mathrm{C}$. Then, requisite amounts of potassium persulfate (KPS) were added. After stirring $10 \mathrm{~min}$, a mixture of monomers (BA/MMA/DAAM) was dropped for 4 hours, and stirred for a further 8 hours at $85^{\circ} \mathrm{C}$. The obtained emulsion was cooled to $50{ }^{\circ} \mathrm{C}$. The target $\mathrm{pH}$ value was adjusted at $7-8$ by adding $10 \% \mathrm{NaOH}$ solution. Then, it was filtered through a filter of 100- mesh sieve. This afforded crosslinkedable PoSt-g-BMD copolymer emulsion.

\subsection{Preparation of self-crosslinkable films}

The self-crosslinkable films were prepared by mixing the crosslinkedable PoSt-g-BMD copolymer with ADH according to the formula ratio. Then, the mixture was stirring for a period of $45 \mathrm{~min}$. PoSt-g-BMD copolymer could react with $\mathrm{ADH}$ at ambient temperature to form the selfcrosslinkable emulsion, which obtained a self-crosslinkable thin film (PoSt-g-BMD+ADH).

\subsection{Preparation of humidity controlling PoSt-g-BMD coatings (PoSt-g-BMD-C)}

The humidity controlling coatings were prepared by the PoSt-g-BMD copolymer emulsion, pigments, fillers, $\mathrm{ADH}$ and water. First, the pigments, fillers, and coating assistants were dispersed in water with stirring. Then, the PoSt-g-BMD copolymer emulsion and ADH were added. The mixture was ground with an attritor filled with glass balls of $0.3 \mathrm{~mm}$ in diameter for a period of $45 \mathrm{~min}$ at room temperature, which afforded the humidity controlling coatings (PoSt-g-BMD-C).

\subsection{Measurements of crosslinkedable PoSt-g-BMD emulsion, film and coatings}

In order to attain a quantitative and qualitative understanding of emulsion polymerization, film and coatings, further information was sought from experiments. To this end, the following measurements were made.

\subsubsection{The conversion (C\%) and graft percentage (PG\%)}

Firstly, Ungrafted polyMMA, polyDAAM and polyBA homopolymers were extracted by dispersing the graft copolymer in acetone that contained sufficient water to impart $25 \%$ moisture to the polymer. This mixture was stirred for 24 hours and was then centrifuged to separate insoluble graft copolymer from acetone soluble homopolymer. Two more extractions with acetone were then carried out. Supernatant and acetone-washed graft copolymer were dried in vacuum drying oven. Then, the residual homopolymers in the dried polymer product was separated by Soxhlet extraction with acetone and then glacial acetic acid for 12 hours. The conversion $(\mathrm{C} \%)$ and graft percentage (PG\%) were calculated through following Equation 1, and 2 respectively. The calculations of $\mathrm{C} \%$ and PG\% were similar to what reported by Meshram et al. ${ }^{23}$ and Song et al. ${ }^{24}$.

Conversion $(\mathrm{C} \%)=\mathrm{w}_{1} / \mathrm{w}_{2} \times 100 \%$

Graft percentage $(\mathrm{PG} \%)=\mathrm{w}_{3} / \mathrm{w}_{1} \times 100 \%$

where $\mathrm{w}_{1}$ is the weight of polymer formed extraction $(\mathrm{g}), \mathrm{w}_{2}$ is the weight of monomer taken $(\mathrm{g})$, and $\mathrm{w}_{3}$ is the weight of starch taken $(\mathrm{g})$.

\subsubsection{Solid content}

A certain quantity of emulsion was cast into a petri dish and dried to a constant weight in a dry oven at $85^{\circ} \mathrm{C}$ for 8 hours. Solid content was calculated by the following Equation 3:

Solid content (wt. \%) $=\left(\mathrm{W}_{\mathrm{DE}} / \mathrm{W}_{\mathrm{E}}\right) \times 100 \%$

where $\mathrm{W}_{\mathrm{DE}}$ is the weight of dry film $(\mathrm{g})$, and $\mathrm{W}_{\mathrm{E}}$ is the weight of polymer emulsion formed extraction $(\mathrm{g})$.

\subsubsection{IR spectroscopy}

Fourier-transform infrared (FT-IR) spectra were recorded with the Digilab FTS3000 spectrometer. One hundred scans were collected for each sample at a resolution of $4 \mathrm{~cm}^{-1}$ over the wavenumber region $4000-400 \mathrm{~cm}^{-1}$. The polymer product was separated by Soxhlet extraction.

\subsubsection{TG analysis}

The thermal gravimetric (TG) analysis was performed with the Pyris Diamond (Perkin Elmer) under the nitrogen atmosphere at a heating rate of $10^{\circ} \mathrm{C} / \mathrm{min}$ from 20 to $720^{\circ} \mathrm{C}$. The polymer product was separated by Soxhlet extraction.

\subsubsection{Scanning electron microscopy (SEM)}

The morphologies of the gold-coated coatings (PoStg-BMD-C) were observed with the scanning electron microscope (SEM), type JSM-6701F.

\subsubsection{Mechanical characterizations of the coatings (PoSt-g-BMD-C)}

For physical and mechanical characterizations, the coatings with a wet thickness of $100 \mu \mathrm{m}$ were cast on glass plates and stainless steel plates, and then they were airdried at room temperature $\left(25^{\circ} \mathrm{C}\right)$ for 48 hours. Important mechanical characterizations were then measured with national standard methods of China, such as, hardness, adhesion, water resistance, drying time and freezing-thawing resistance.

\section{Results and Discussion}

Starches are potential sources for a wide variety of polymers as they are renewable and ecologically friendly. Particular attention has been attached to synthesis of starch graft copolymer due to an increasing interest in the synthesis of new cost-effective polymer ${ }^{25}$. Cross-linking is a common approach to improve the performance of starch for various applications. Starch and starch products have been cross- 
linked with cross-linking agents to improve the mechanical properties and water stability of starch products ${ }^{26}$.

Here, the first goal was to prepare crosslinkable PoSt-based graft copolymer (PoSt-g-BMD) that might serve as waterborne coatings, which can be cured at ambient temperature and possess good properties. The graft copolymerization was represented in Figure 1. In order to improve the properties of target coatings, PoSt-g-BMD containing diacetone acrylamide (DAAM) as functional monomer and adipic acid dihydrazide (ADH) as cross-linker, could cure fast at ambient temperature during the preparation of paint film or coatings (Figure 2). The reaction results were that form hydrazones moieties and form the cross-link points within the film, and the cross-linked films showed excellent water resistance and solvent resistance.

\subsection{Conversion (C\%) and graft percentage (PG\%) of PoSt-g-BMD}

We found that conversion of monomers and graft percentage of PoSt were influenced by proportions of raw material, initiator, reaction temperature and time. These influences were investigated as follows:

\subsubsection{The effect of proportions of raw materials on $\mathrm{C} \%$ and $\mathrm{PG} \%$}

The effect of feeding ratio on $\mathrm{C} \%$ and $\mathrm{PG} \%$ was measured at $85^{\circ} \mathrm{C}$. The results were shown in Figure 3 . When ratio of PoSt to mixed monomers was less than 1:5, the monomer conversion was opposite to the graft percentage. The maximum of graft percentage (PG\%) was $80 \%$ when w (PoSt) / w (Monomers) were up to 1:4. However, a further increase of the PoSt concentration had greatly reduced the $\mathrm{C} \%$ and $\mathrm{PG} \%$.

\subsubsection{Effect of w (BA)/ w (MMA) on C\% and PG\%}

The ratio of BA to MMA also influenced the properties of the copolymer emulsion. It was not only related to grafting percentage, but also affected the monomer conversion (Figure 4). It showed that $\mathrm{C} \%$ and $\mathrm{PG} \%$ increased with the increasing of the BA concentration. When the mass ratio of

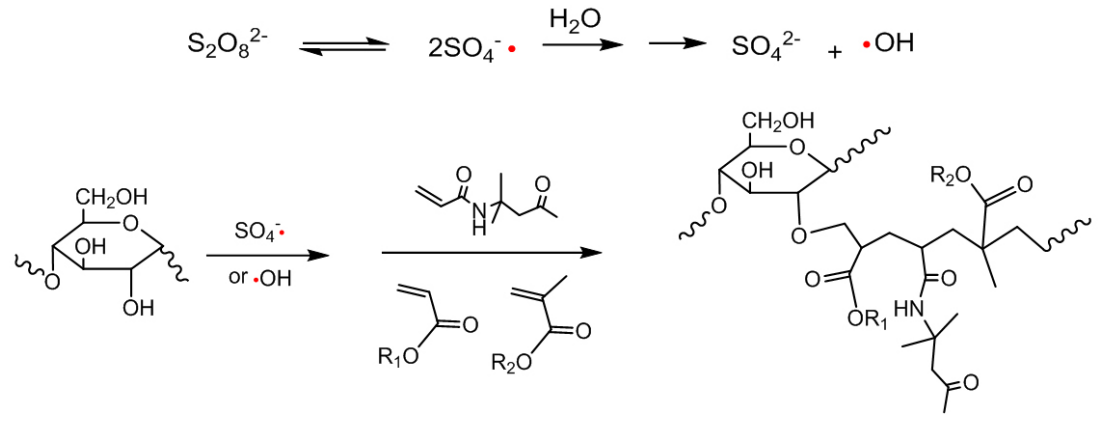

PoSt

PoSt-g-BMD

$\mathrm{R}_{1}=\mathrm{C}_{4} \mathrm{H}_{9}, \quad \mathrm{R}_{2}=\mathrm{CH}_{3}$

Figure 1. The graft copolymerization of BA, MMA and DAAM on PoSt $\left(\mathrm{R}_{1}=\mathrm{C}_{4} \mathrm{H}_{9}, \mathrm{R}_{2}=\mathrm{CH}_{3}\right)$.
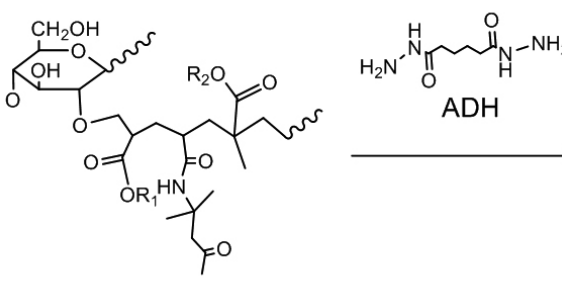

PoSt-g-BMD

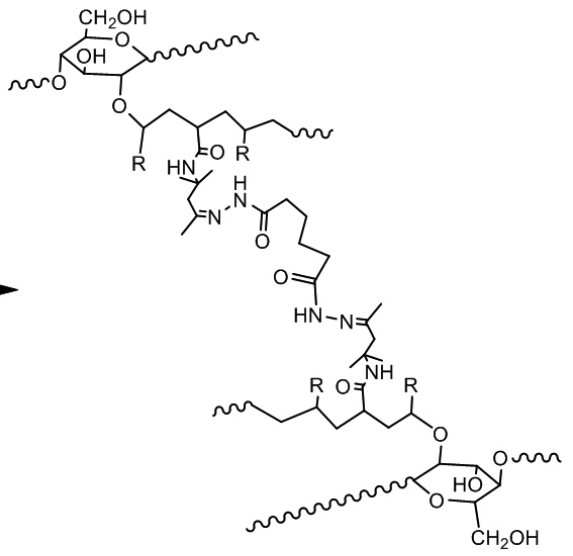

PoSt-g-BMD + ADH

Figure 2. Cross-linking reaction of PoSt-g-BMD with ADH. 
BA to MMA was $1: 1$, the conversion reached maximum. However, the mixed monomers (BA/MMA) concentration increased over $1: 1$, both the $\mathrm{C} \%$ and $\mathrm{PG} \%$ decreased with the increasing in BA of the monomers. This may be ascribed to the following facts. On the one hand, increasing the monomer concentration was beneficial to the diffusion of monomer molecules on the backbone, thus leading to an increasing in $\mathrm{PG} \%$ and $\mathrm{C} \%$. On the other hand, the high monomer concentrations were good for the formation of accept-donator complexes between PoSt and monomers, which led to the grafting process ${ }^{20,24,26}$. Therefore, an optimum soft/hard monomer concentration of this system was $1: 1$.

\subsubsection{The effect of KPS content on $\mathrm{C} \%$ and $\mathrm{PG} \%$}

The PoSt-g-BMD copolymers were prepared with KPS as initiator at five different concentrations (0.5, 1.0, $1.5,2.0$ and 2.5 wt. $\%$ of the PoSt) at $85^{\circ} \mathrm{C}$ for 8 hours. And the effect of KPS content on $\mathrm{C} \%$ and $\mathrm{PG} \%$ was shown in Figure 5. The conversion increased with the increasing KPS concentrations and up to a maximum at $1.0 \mathrm{wt} \% \mathrm{KPS}$.
However, the $\mathrm{C} \%$ and $\mathrm{PG} \%$ both decreased with further increases in the KPS concentration above $1.50 \mathrm{wt} . \%$, so the optimum values observed were between 1 and $1.50 \mathrm{wt} . \%$. The higher concentrations of KPS produced the higher monomers conversion; but when the KPS concentration reached $2.0 \mathrm{wt} . \%$, chain transfer or homopolymer formation was causing lower monomer $\mathrm{C} \%$ and $\mathrm{PG} \%$.

\subsubsection{The effect of reaction temperature on $\mathrm{C} \%$ and $\mathrm{PG} \%$}

Reaction temperature also played an important role in graft copolymerization. Figure 6 showed the PoSt-g-BMD copolymer were prepared from three different reaction temperatures $\left(70,80\right.$ and $\left.90{ }^{\circ} \mathrm{C}\right)$ with $\mathrm{w}$ (starch) / w (monomers) ratio of $1: 4$, KPS at 1 wt. $\%$ for 8 hours. The conversion increased with the increasing of reaction temperature in the range of $70-80{ }^{\circ} \mathrm{C}$. And then conversion decreased gradually when temperature higher than $80{ }^{\circ} \mathrm{C}$. But, the GP\% also increased with increasing reaction temperatures over $80{ }^{\circ} \mathrm{C}$. And it reached a maximum when the reaction temperature was $90^{\circ} \mathrm{C}$ because rate of

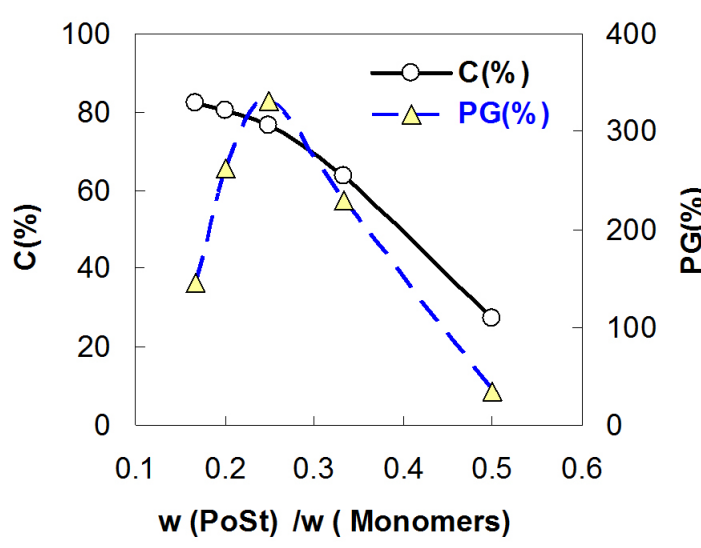

Figure 3. The effect of proportions of raw materials on $\mathrm{C} \%$ and PG\% (Cond.: w(BA) / w(MMA) = 1: 1, KPS: 1.50 wt. $\%$, Temp: $85^{\circ} \mathrm{C}$, Time: 8 hours).

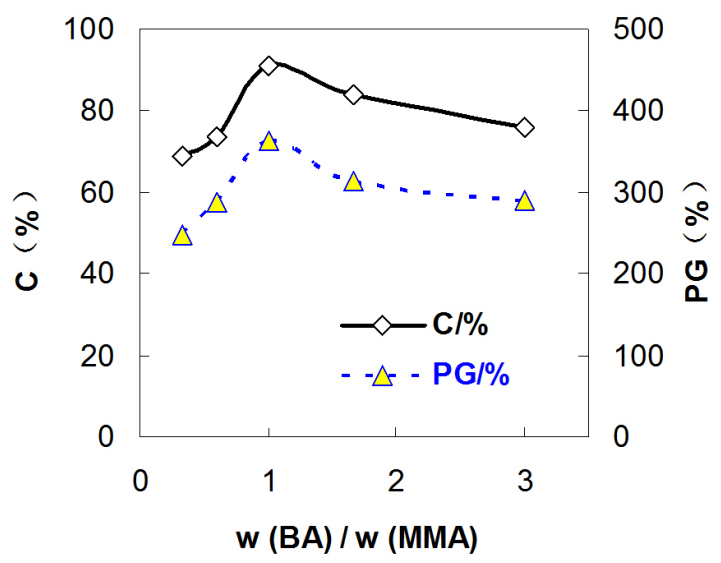

Figure 4. Effect of $\mathrm{w}(\mathrm{BA}) / \mathrm{w}(\mathrm{MMA})$ on $\mathrm{C} \%$ and $\mathrm{PG} \%$ (Cond.: $\mathrm{w}($ PoSt $) / \mathrm{w}($ Monomers $)=1: 4$, KPS: $1.50 \mathrm{wt} . \%$, Temp: $85^{\circ} \mathrm{C}$, Time: 8 hours).

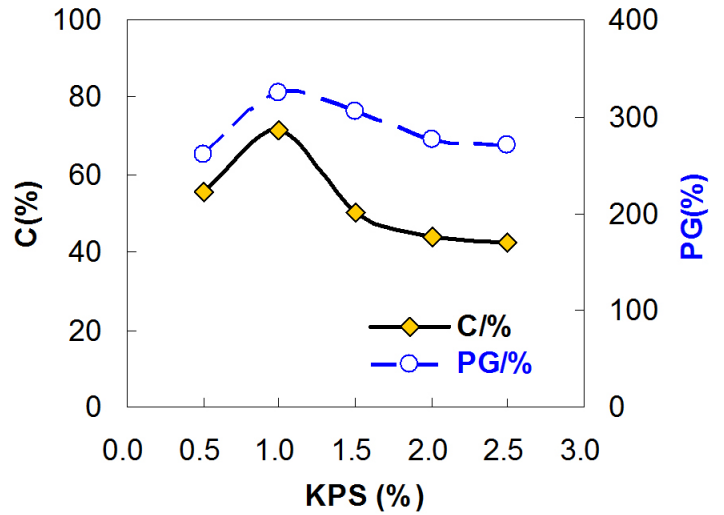

Figure 5. The effect of KPS content on $\mathrm{C} \%$ and PG\% (Cond.: w $(\mathrm{BA}) / \mathrm{w}(\mathrm{MMA})=1: 1, \mathrm{w}(\mathrm{PoSt}) / \mathrm{w}($ Monomers $)=1: 4$, Temp: $85^{\circ} \mathrm{C}$, Time: 8 hours).

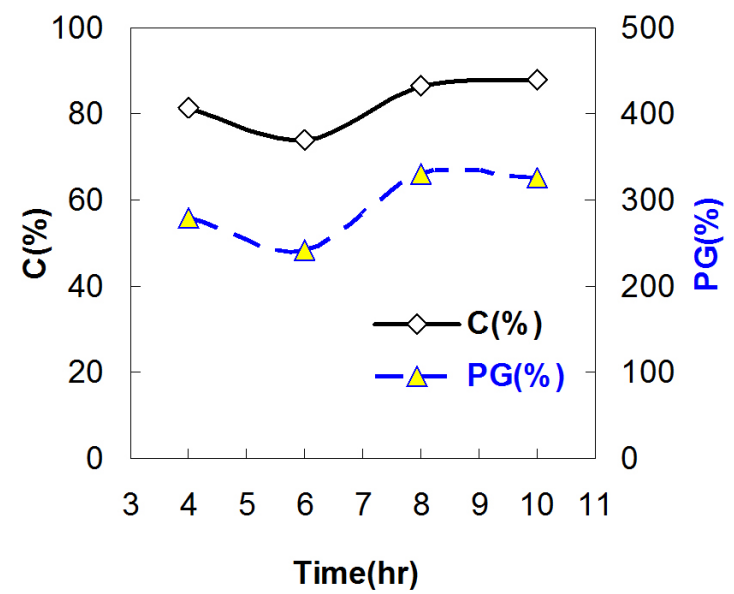

Figure 6. The effect of reaction temperature on $\mathrm{C} \%$ and $\mathrm{PG} \%$ (Cond.: $\mathrm{w}(\mathrm{BA}) / \mathrm{w}(\mathrm{MMA})=1: 1, \mathrm{w}($ PoSt $) / \mathrm{w}($ Monomers $)=1: 4, \mathrm{KPS}$ : 1.50 wt. $\%$, Time: 8 hours). 
homopolymerization was increased above $90^{\circ} \mathrm{C}$. Therefore, the optimum reaction temperature was $85^{\circ} \mathrm{C}$ in this system.

\subsubsection{The effect of reaction time on $\mathrm{C} \%$ and $\mathrm{PG} \%$}

The effect of reaction time on $\mathrm{C} \%$ and $\mathrm{PG} \%$ of PoSt-g-BMD copolymer was determined in the range of 2-10 hours in Figure 7. It was found that $\mathrm{C} \%$ and $\mathrm{PG} \%$ were the highest at 8 hours. Because graft copolymerization reacted only on the surface of the starch granules, once the starch surface was fully covered with BA/MMA/DAAM, no more new BA/MMA/DAAM could attached on the starch. As a result, the reaction time longer than 8 hours that generated more BA/MMA/DAAM homopolymer and the emulsion viscosity increased. Therefore, the optimum reaction time was 8 hours in this system.

\subsection{Structure and properties of PoSt-g-BMD emulsion and coatings}

\subsubsection{FT-IR spectra}

The FT-IR spectra of the PoSt, extracted PoSt-g-BMD copolymer and PoSt-g-BMD+ADH film were shown in Figure 8. Some characteristic peaks of starch, such as $-\mathrm{OH}$

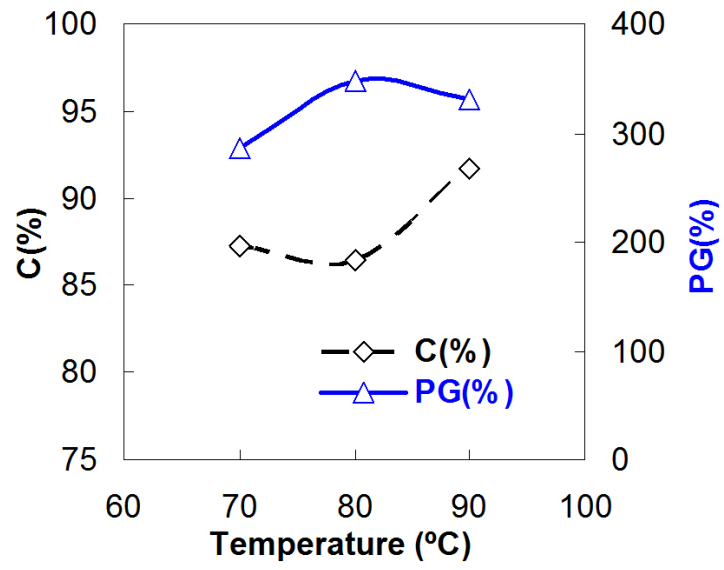

Figure 7. The effect of reaction time on $\mathrm{C} \%$ and $\mathrm{PG} \%$ (Cond.: $\mathrm{w}(\mathrm{BA}) / \mathrm{w}(\mathrm{MMA})=1: 1, \mathrm{w}(\mathrm{PoSt}) / \mathrm{w}($ Monomers $)=1: 4, \mathrm{KPS}$ : 1.50 wt. $\%$, Temp: $\left.85^{\circ} \mathrm{C}\right)$.

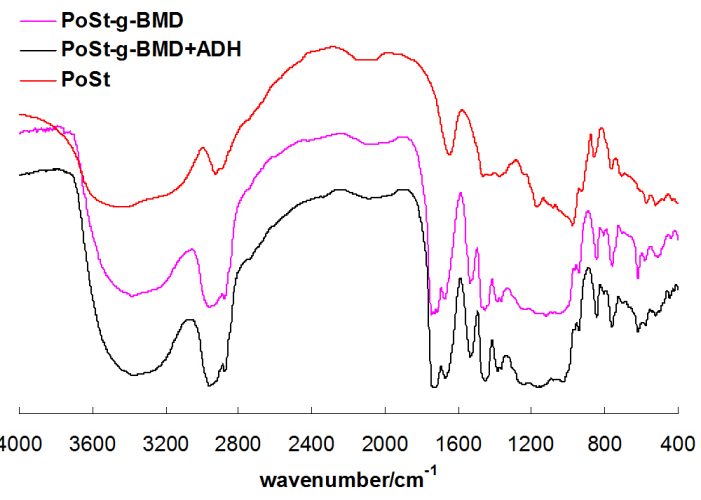

Figure 8. FT-IR spectra of PoSt, PoSt-g-BMD and PoSt-g$\mathrm{BMD}+\mathrm{ADH}$ copolymer. stretching vibration and $\mathrm{C}-\mathrm{O}$ stretching vibration appeared at $3600 \mathrm{~cm}^{-1}$ and $1647 \mathrm{~cm}^{-1}$. The peak appeared near $1530 \mathrm{~cm}^{-1}, 1545 \mathrm{~cm}^{-1}$ and $1734 \mathrm{~cm}^{-1}$ in the PoSt-g-BMD could be attributed to the $-\mathrm{OH}$ bending vibration, $\mathrm{C}=\mathrm{O}$ stretching vibration and $\mathrm{COO}^{-}$symmetric stretching vibration. However, the N-H stretching vibration of this amide group appeared at $3511 \mathrm{~cm}^{-1}$ and overlapped with the broad bands of - $\mathrm{OH}$ vibrations. The amide group in the PoSt-g-BMD indicated that DAAM had been successfully copolymerized. The characteristic absorption of the $\mathrm{C}=\mathrm{C}$ bond at $1640 \mathrm{~cm}^{-1}$ and the $=\mathrm{C}-\mathrm{H}$ bond at $1423 \mathrm{~cm}^{-1}$ in DAAM disappeared; it indicated that the monomers had polymerized. Moreover, ADH of the $-\mathrm{N}-\mathrm{H}\left(\mathrm{NH}_{2}\right)$ stretching vibration at $3319 \mathrm{~cm}^{-1}$ and DAAM of ketone carbonyl $(-\mathrm{C}=\mathrm{O}-)$ stretching vibration at $1747 \mathrm{~cm}^{-1}$ all disappeared, which indicated that DAAM cross-linked with $\mathrm{ADH}$ at ambient temperature.

\subsubsection{Thermo gravimetric analysis (TGA)}

The thermo gravimetric curves of PoSt, extracted PoStg-BMD copolymer and PoSt-g-BMD+ADH film were shown in Figure 9. The curves of PoSt, PoSt-g-BMD and PoSt-g-BMD+ADH showed that their first weight loss in the range of $20^{\circ} \mathrm{C}$ to $180{ }^{\circ} \mathrm{C}$ was due to the loss of physically absorbed interlayer water. It can be seen the PoSt began to decompose at $280{ }^{\circ} \mathrm{C}$ and decomposed completely at $495^{\circ} \mathrm{C}$. However, the PoSt-g-BMD and PoSt-g-BMD+ADH began to decompose at $400{ }^{\circ} \mathrm{C}$ and decomposed completely at $520{ }^{\circ} \mathrm{C}$. It can be concluded that PoSt-g-BMD grafting copolymer emulsion and PoSt-g-BMD+ADH grafting copolymer had much higher thermal stability than pure PoSt.

\subsubsection{The basic properties of the PoSt-g-BMD copolymer}

The PoSt-g-BMD copolymer would be used in architectural indoor coatings. Moreover, the mechanical stability, viscosity, solid content, drying time, freezingthawing resistance ( 3 times) and storage stability ( 3 months) of the emulsion reached the standards of Chinese indoor coating standards of synthetic emulsion(GB/T172079(89)). Then, some important mechanical parameters were measured by national standard methods of china, such as hardness test (GB/T 1730-93), adhesion test, gloss test(GB 9754-88), and water resistance(GB/T1733-93) It

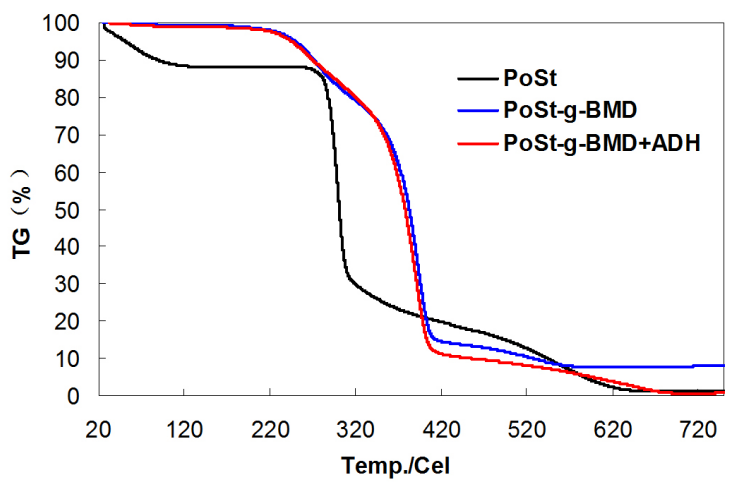

Figure 9. Thermo gravimetric analysis (TGA) of PoSt, PoSt-g$\mathrm{BMD}$ and PoSt-g-BMD+ADH copolymer. 


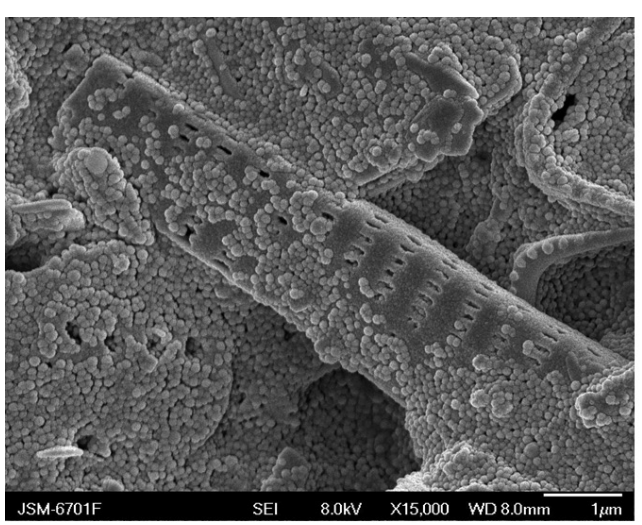

(a)

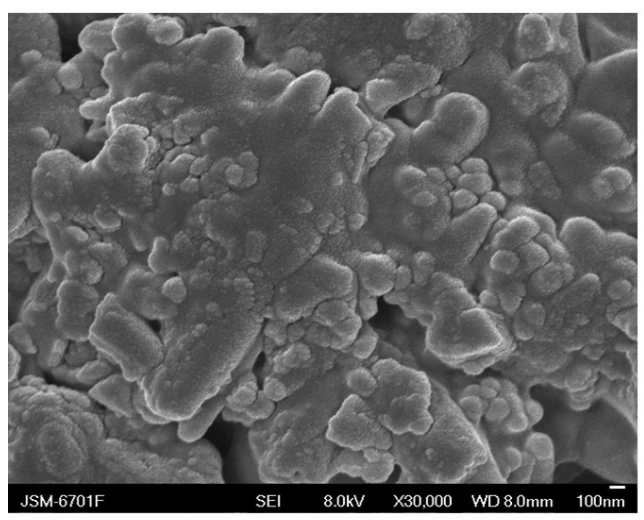

(c)

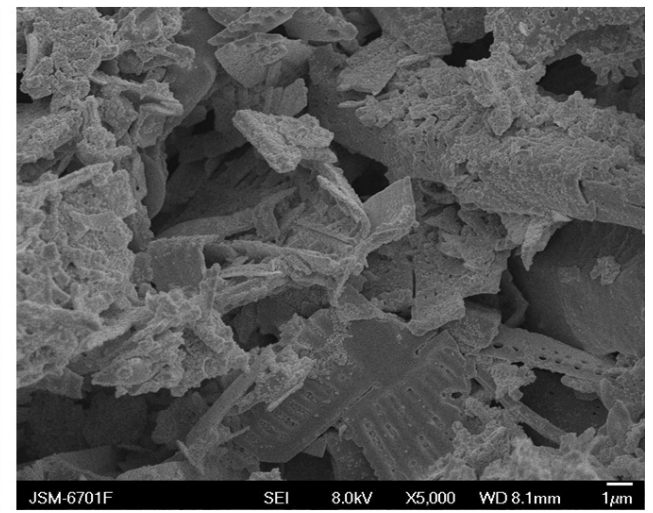

(b)

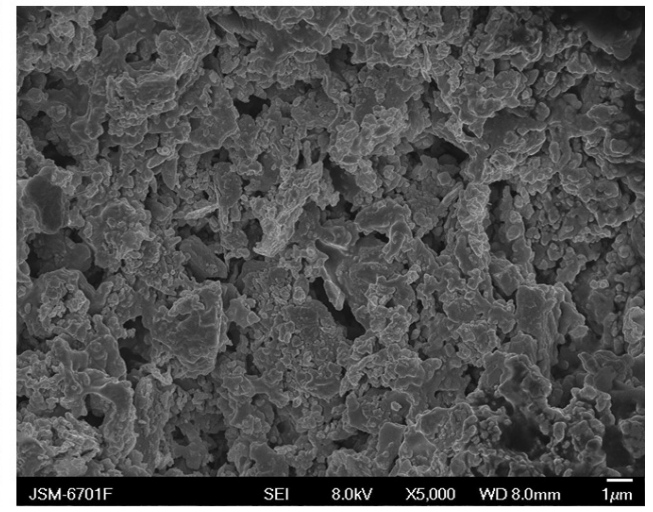

(d)

Figure 10. SEM images of PoSt-g-BMD-C and traditional interior wall coatings ((a) Surface of PoSt-g-BMD-C; (b) Cross Profile of PoSt-g-BMD-C; (c) Surface of traditional interior wall coatings; (d) Cross Profile of Traditional interior wall coatings).

showed that the basic properties of the humidity-sensitive coatings had reached the standard of Chinese interior wall coatings standards.

\subsubsection{The basic properties of the PoSt-g-BMD-C}

The PoSt-g-BMD-C was prepared by the PoSt-gBMD copolymer, pigments, fillers, $\mathrm{ADH}$ and water. The coatings with a wet thickness of $100 \mu \mathrm{m}$ were cast on glass plates and stainless steel plates, and air-dried at room temperature $\left(23 \pm 2{ }^{\circ} \mathrm{C}\right)$ for 48 hours for physical and mechanical characterizations. Then, some important mechanical characterizations were measured by national standard methods of China, such as contrast ratio, hardness test (GB/T 1730-93), adhesion test (GB/T1720-79(89)) and water resistance (GB/T1733-93). It showed that the basic properties of the humidity-controlling coatings had reached the standard of Chinese interior wall coatings standards.

\subsubsection{The morphology of the PoSt-g-BMD-C coatings}

In order to verify the suggested humidity controlling mechanism of PoSt-g-BMD-C, the surface and crosssection morphologies of the crosslinkable humidity controlling PoSt-g-BMD coatings (PoSt-g-BMD-C) and traditional interior wall coatings were measured by scanning electron microscope (SEM) in Figure 10. As shown in Figure 10a, b, the surface attached starch particles and cross-section of PoSt-g-BMD-C presented lots of holes and voids in microstructure. Furthermore, it indicated that the coating surface was loose as coralliform. This meant that its specific surface area was large, which contained more porous channels and interstice. Thus, water, moisture, and gas could pass through the coating freely. And it was also a proof that it has good water-absorption ${ }^{4}$. The PoSt-g-BMD-C had good water absorption, high water absorption speed, and good water and gas permeability. The morphologies of traditional interior wall coating were shown in Figure 10c, d. This coating surface was more compact due to its gravel accumulation. Its specific surface area was low, which contained less-porous channels and interstice. Thus, its water and gas permeability was poorer than the humidity controlling coatings. The reason was that the humidity controlling coatings were composed of the crosslinkable PoSt-g-BMD copolymer emulsion, which added porous and multilayer fillers. The filler particles were parceled by PoSt-g-BMD grafting copolymer. They were all water-absorbent materials. Porous filler with a huge surface 


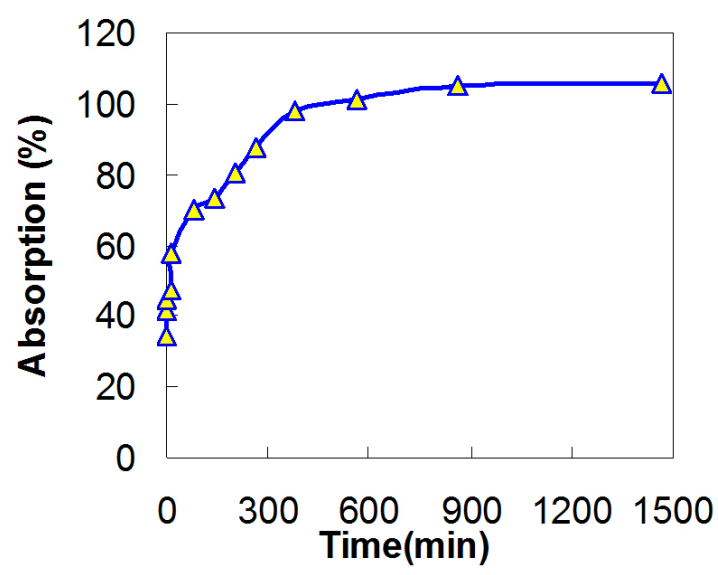

Figure 11. Water absorption rate of PoSt-g-BMD-C.

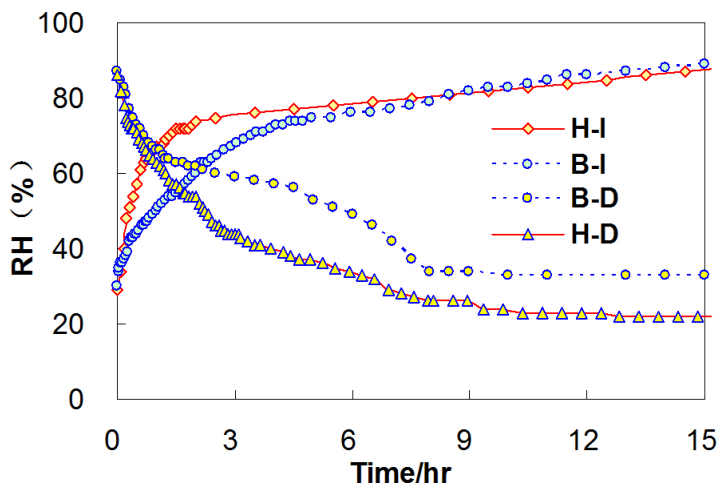

Figure 12. The dehumidifying and humidity increasing properties of the PoSt-g-BMD-C (H-I: Humidity increasing with moist coatings, B-I: Blank humidity increase, H-D: Humidity decreasing with dry coatings; B-D: Blank without coatings).

area and pore volume afforded expansion space for volume expansion of PoSt-g-BMD grafting copolymer. Therefore, the PoSt-g-BMD-C with the better performance of humidity controlling and PoSt was a natural raw material.

\subsection{Water absorption and humidity controlling function of PoSt-g-BMD coatings}

\subsubsection{Water absorption properties of the PoSt-g-BMD-C}

The coatings were cast on glass plates with a wet thickness of $100 \mu \mathrm{m}$, and they were air-dried at room temperature $\left(23 \pm 2{ }^{\circ} \mathrm{C}\right)$ for 48 hours, and then weighed the glass plate and sample. Then dipped into the distilled water at $23 \pm 2{ }^{\circ} \mathrm{C}$ and weighed at regular intervals. The water absorption and water absorption rate of PoSt-g-BMD coatings were measured. In Figure 11, it indicated that the water absorption rate of PoSt-g-BMD coatings was very fast, which got to $80 \%$ about $15 \mathrm{~min}$, and was close to be saturated about 9 hours, which could get to $105 \%$. Meanwhile, during adsorbing water, the thickness of the PoSt-g-BMD-C had no change as the porous and multi-layer filler with a huge surface area and pore volume afforded expansion space for volume expansion of humidity controlling coatings.

\subsubsection{The humidity controlling behaviors of PoSt-g- BMD-C}

The humidity controlling properties of PoSt-g-BMD coating were investigated in an artificial climate box according to reference Wang et al. ${ }^{6}$, Song et al. ${ }^{7}$. The dehumidifying activity of the humidity controlling coatings was measured. In Figure 12, the curve H-D showed dehumidifying activity of humidity controlling coatings. The blank (or control) experiment (B-D) was measured by withdrawing the coated sheet. The results showed that the relative humidity $(\mathrm{RH})$ decreased from $90 \%$ to $40 \%$ within 3 hours. It could keep RH below $30 \%$ for more than 8 hours. However, it needed 7 hours for decreasing $\mathrm{RH}$ from $90 \%$ to $40 \%$ if the box was empty. It was concluded that the humidity-sensitive coatings showed excellent dehumidification. Meanwhile, humidification of the humidity controlling coatings was measured. A piece of coated sheet whose area equaled with that of the glass vessel, which had been saturated by water, was placed in the artificial climate box which $\mathrm{RH}$ was below $30 \%$. H-I is the curve of humidity increasing activity of the humidity controlling coatings. The blank experiment (B-I) was measured by replacing the coated sheet with a dish-like glass vessel (diameter: $15 \mathrm{~cm}$ ) which was filled with water. The results showed that the RH increased over $60 \%$ within 1.2 hours by water saturated coated sheet, which was similar to keeping water in it. That meant the humidity controlling property of coatings prepared by crosslinkable PoSt-gBMD-C was excellent. And it could be used in interior walls for humidity controlling.

\section{Conclusions}

The crosslinkable PoSt-based copolymer emulsion (PoSt-g-BMD) was synthesized by graft copolymerization. The grafting polymers onto starch were confirmed by both FT-IR and TGA. The PoSt-g-BMD containing diacetone acrylamide (DAAM) as functional monomer and adipic acid dihydrazide (ADH) as cross-linker, could cure fast at ambient temperature during the preparation of paint film or coatings. The emulsion was used in interior wall coatings for controlling humidity, and could increase indoor humidity or dehumidify in dry or moist environments. The obtained coatings had good humidity-sensitivity and humidity-retention. This emulsion is a promising candidate for indoor wall coatings which could be widely used to control humidity.

\section{Acknowledgements}

The authors are grateful to the NSFC (21263024, 21244003), PCSIRT(IRT1177), the Gansu Sci \& Techn Support Project (1011GKCA017), Res Fund for Postgraduate Tutors in Inst Higher Edu Gansu Prov China (1201-15) for financial support. 


\section{References}

1. Kalamees T, Korpi M, Vinha J and Kurnitski J. The effects of ventilation systems and building fabric on the stability of indoor temperature and humidity in Finnish detached houses. Building and Environment. 2009; 44(8):1643-1650. http:// dx.doi.org/10.1016/j.buildenv.2008.10.010

2. LvWH,WangRM,He YFandZhangHF.Preparationandapplication of smart coatings. Progress in Chemistry. 2008; 20(10):351-361.

3. Mahmouda KR, Al-Sigenya S, Sharshara T and El-Hamshary $\mathrm{H}$. Positron annihilation study on free volume of amino acid modified, starch-grafted acrylamide copolymer. Radiation Physics and Chemistry. 2006; 75(5):590-595. http://dx.doi. org/10.1016/j.radphyschem.2005.12.037

4. Wang RM, Wang JF, Wang XW, He YF, Zhu YF and Jiang ML. Preparation of acrylate-based copolymer emulsion and its humidity controlling mechanism in interior wall coatings. Progress in Organic Coatings. 2011; 71(4):369-375. http:// dx.doi.org/10.1016/j.porgcoat.2011.04.007

5. Woloszyna M, Kalamees T, Abadie MO, Steeman M and Kalagasidis AS. The effect of combining a relative-humiditysensitive ventilation system with the moisture-buffering capacity of materials on indoor climate and energy efficiency of buildings. Building and Environment. 2009; 44(3):515-524. http://dx.doi.org/10.1016/j.buildenv.2008.04.017

6. Wang RM, Wang JF, Lv WH, Guo JF, He YF and Jiang ML. Preparation of amphiphilic acrylic acid copolymer and its application in humidity-sensitive coatings. Journal of Applied Polymer Science. 2011; 120(5):2497-3117. http://dx.doi. org/10.1002/app.33473

7. Song $\mathrm{H}, \mathrm{Wu} \mathrm{D}$, Zhang RQ, Qiao LY, Zhang S, Lin $\mathrm{H}$ et al. Synthesis and application of amphoteric starch graft polymer. Carbohydrate Polymers. 2009; 78(2):253-257. http://dx.doi. org/10.1016/j.carbpol.2009.03.027

8. Wang RM, Lv WH, He YF, Wang Y and Guo JF. An emulsifierfree core-shell polyacrylate/diacetone acrylamide emulsion with nano- $\mathrm{SiO}_{2}$ for room temperature curable waterborne coatings. Polymers for Advanced Technologies. 2010; 21(2):128-134.

9. Chung YL, Ansari S, Estevez L, Hayrapetyan S, Giannelis EP and Lai HM. Preparation and properties of biodegradable starch-clay nanocomposites. Carbohydrate Polymers. 2010; 79(2):391-396. http://dx.doi.org/10.1016/j. carbpol.2009.08.021

10. Dragan E, Apopei DF. Synthesis and swelling behavior of $\mathrm{pH}-$ sensitive semi-interpenetrating polymer network composite hydrogels based on native and modified potatoes starch as potential sorbent for cationic dyes. Chemical Engineering Journal. 2011; 178: 252-263. http://dx.doi.org/10.1016/j. cej.2011.10.066

11. Teramoto N,Motoyama T, Yosomiya R and Shibata M. Synthesis, thermal properties, and biodegradability of propyl-etherified starch. European Polymer Journal. 2003; 39(2):255-261. http:// dx.doi.org/10.1016/S0014-3057(02)00199-4

12. Jiang YX, Ju BZ, Zhang SF and Yang JZ, Preparation and application of a new cationic starch ether-Starchmethylene dimethylamine hydrochloride. Carbohydrate Polymers. 2010; 80(2):467-473. http://dx.doi.org/10.1016/j. carbpol.2009.12.002

13. Zhou J, Ren LL, Tong J, Xie L and Liu ZQ. Surface esterification of corn starch films: Reaction with dodecenyl succinic anhydride. Carbohydrate Polymers. 2009; 78(4):888-893. http://dx.doi.org/10.1016/j.carbpol.2009.07.017
14. Ruan H, Chen QH, Fu ML, Xu Q and He GQ. Preparation and properties of octenyl succinic anhydride modified PoSt. Food Chemistry. 2009; 114(1):81-86. http://dx.doi.org/10.1016/j. foodchem.2008.09.019

15. Acquarone VM and Rao MA. Influence of sucrose on the rheology and granule size of cross-linked waxy maize starch dispersions heated at two temperatures. Carbohydrate Polymers. 2003; 51(4):451-458. http://dx.doi.org/10.1016/ S0144-8617(02)00217-5

16. Wurzburg OB. Nutritional aspects and safety of modified food starches. Nutrition Reviews. 1986; 44(2):74-79. PMid:3703392. http://dx.doi.org/10.1111/j.1753-4887.1986.tb07590.x

17. Hirsch JB, and Kokini JL. Understanding the Mechanism of Cross-Linking Agents ( $\mathrm{POCl}_{3}$, STMP, and EPI) Through Swelling Behavior and Pasting Properties of Cross-Linked Waxy Maize Starches. Cereal Chemistry. 2002; 79(1):102-107. http://dx.doi.org/10.1094/CCHEM.2002.79.1.102

18. Saboktakin MR, Maharramov A and Ramazanov MA $\mathrm{pH}$-sensitive starch hydrogels via free radical graft copolymerization, synthesis and properties. Carbohydrate Polymers. 2009; 77(3):634-638. http://dx.doi.org/10.1016/j. carbpol.2009.02.004

19. Kaewtati K and Tanrattanakul V. Preparation of cassava starch grafted with polystyrene by suspension polymerization. Carbohydrate Polymers. 2008; 73(4):647-655. http://dx.doi org/10.1016/j.carbpol.2008.01.006

20. Athawale VD and Vidyagauri VL. Graft copolymerization onto starch. II. Grafting of acrylic acid and preparation of it's hydrogels. Carbohydrate Polymers. 1998; 35(1-2):21-27. http:// dx.doi.org/10.1016/S0144-8617(97)00138-0

21. Tessier R, Lafranche E and Krawczak P. Development of novel melt-compounded starch-grafted polypropylene/ polypropylene-grafted maleic anhydride/organoclay ternary hybrids. Polymer Letters. 2012; 6(11):937-952. http://dx.doi. org/10.3144/expresspolymlett.2012.99

22. Ma XF, Chang PR, Yu JG and Wang N. Preparation and properties of biodegradable poly(propylene carbonate)/ thermoplastic dried starch composites. Carbohydrate Polymers. 2008; 71(2):229-234. http://dx.doi.org/10.1016/j carbpol.2007.05.033

23. Qudsieh IYM, Fakhru'l-Razi A, Muyibi SA, Ahmad MB, Rahman MZA and WMZW Yunus. Preparation and characterization of poly(methyl methacrylate) grafted sago starch using potassium persulfate as redox initiator. Journal of Applied Polymer Science. 2004; 94(5):1891-1897. http:// dx.doi.org/10.1002/app.20883

24. Song H, Zhang SF, Ma XC, Wang DZ and Yang JZ, Synthesis and application of starch-graft-poly(AM-co-AMPS) by using a complex initiation system of CS-APS. Carbohydrate Polymers. 2007; 69(1):189-195. http://dx.doi.org/10.1016/j. carbpol.2006.09.022

25. Bemiller JN. Starch Modification: Challenges and Prospects Starch. Starch - Stärke. 1997; 49(4):127-131. http://dx.doi org/10.1002/star.19970490402

26. Singh B and Sharma N. Optimized synthesis and characterization of polystyrene graft copolymers and preliminary assessment of their biodegradability and application in water pollution alleviation technologies. Polymer Degradation and Stability. 2007; 92(5):876-885. http://dx.doi. org/10.1016/j.polymdegradstab.2007.01.019 